Please do not remove this page

RMIT

UNIVERSITY

\title{
Adulterants and altruism: A qualitative investigation of "drug checkers" in North America
}

Palamar, Joseph; Acosta, Patricia; Sutherland, Rachel; Shedlin, Michele; Barratt, Monica

https://researchrepository.rmit.edu.au/esploro/outputs/9921863590301341/filesAndLinks?institution=61RMIT_INST\&index=null

Palamar, J., Acosta, P., Sutherland, R., Shedlin, M., \& Barratt, M. (2019). Adulterants and altruism: A qualitative investigation of "drug checkers" in North America. International Journal of Drug Policy, 74, 160-169. https://doi.org/10.1016/j.drugpo.2019.09.017

Document Version: Accepted Manuscript

Published Version: https://doi.org/10.1016/j.drugpo.2019.09.017

Repository homepage: https://researchrepository.rmit.edu.au

CC BY-NC-ND V4.0

(C) 2019 Elsevier B.V. All rights reserved.

Downloaded On 2023/04/26 22:16:42 +1000 
Running head: DRUG CHECKING IN NORTH AMERICA

Word Count: 9,067

Tables: 1

Figures: 0

Adulterants and Altruism: A Qualitative Investigation of "Drug Checkers" in North America

Joseph J. Palamar, ${ }^{1,2}$ Patricia Acosta, ${ }^{1}$ Rachel Sutherland, ${ }^{3}$ Michele G. Shedlin, ${ }^{2}$ and Monica J. Barratt ${ }^{4,3}$

${ }^{1}$ Department of Population Health, New York University School of Medicine, New York, NY, US

${ }^{2}$ Center for Drug Use and HIV/HCV Research, NYU College of Global Public Health, New York, NY, US

${ }^{3}$ National Drug and Alcohol Research Centre, UNSW Sydney, Sydney, Australia

${ }^{4}$ Social and Global Studies Centre, RMIT University, Melbourne, Australia

Address correspondence to: Joseph J. Palamar, Department of Population Health, 180 Madison Avenue, Room 1752, New York, NY 10016 (USA), e-mail:

joseph.palamar@nyulangone.org,

T: 1-646-501-3555. Declaration of Interest: The authors declare no conflict of interest. 


\begin{abstract}
Background: "Drug checking" has become a common harm reduction method used to test illicit substances, such as ecstasy, for purity and/or the presence of adulterants. Formal drug-checking services have been operating for decades, and the use of personal reagent test kits appears to be relatively common; however, little attention has been devoted to understanding the role and broader experiences of 'drug-checkers' (i.e., people who test their own and/or other people's substances). As such, it remains unknown who is engaging in this practice, their motivations for drug-checking, and what barriers they may experience. We addressed this research gap by interviewing people who check drugs about their experiences, with a goal of better understanding drug checking practices.

Methods: We conducted in-depth interviews with 32 adults in North America who reported testing drugs. Coding was conducted in an inductive manner and thematic analysis was used to identify relevant themes.
\end{abstract}

Results: Over half $(56.2 \%)$ of our sample was affiliated with a drug checking organization. Among non-affiliated checkers (43.8\%), the majority (57.1\%) tested for friends, $21.4 \%$ tested only for themselves, and $21.4 \%$ were people who sold drugs and tested for their clients. Motivations were driven largely by altruism, described by checkers as wanting to protect their peers from exposure to adulterants. People interviewed who sold drugs were altruistic in the same manner. Barriers to checking-particularly at nightclubs and festivals_included perceived illegality of test kits and denied approval to test drugs at venues, although many checkers circumvented this barrier by checking drugs without such approval.

Conclusions: Drug checkers in North America seek to educate people who use drugs about the risk of exposure to unexpected substance types, but they face various barriers. Policy change could help ensure that these potentially life-saving services can be provided without fear of fines and/or criminal prosecution.

Keywords: drug checking; harm reduction; ecstasy; new psychoactive substances; altruism 


\section{Introduction}

The production, distribution, and preparation of prohibited drugs are confounded by a lack of quality control and assurance, and sterile production (Cole et al., 2011). Subsequently, illicit drugs carry the risk of containing other substances, in addition to the purported active ingredient. These additional substances are typically classified as 'adulterants' (pharmacologically active ingredients), 'contaminants' (by-products of the manufacturing process), or 'diluents' (inert substances) (Cole et al., 2011). Of these, adulterants are of particular concern, as they appear to have the greatest potential to result in adverse health consequences.

The adulteration of illicit drugs is not a new phenomenon, with evidence of it occurring as early as the 1930s (Morgan, 1982). Ecstasy, in particular, has a long history of adulteration (Hayner, 2002; Morelato et al., 2014; Verweij, 1992) which has been exacerbated in recent years by the emergence of new psychoactive substances (NPS; $~ 900$ identified to date (United Nations Office on Drugs and Crime, 2019). The composition of drugs sold as MDMA/Ecstasy/Molly in particular varies substantially over time and across countries (Brunt et al., 2017)-currently, adulterated ecstasy remains a concern in North America and Australia (primarily among nightclub and dance festival attendees; Australian Criminal Intelligence Commission, 2019; Mohr et al., 2018; Palamar et al., 2017), while high dose/purity ecstasy is dominating the European market (European Monitoring Centre for Drugs and Drug Addiction, 2019). Drugs adulterated with fentanyl and its analogs are now of particular concern in the US as tens of thousands of people are now dying in the US per year from fentanyl exposure (Scholl et al., 2018) and it is likely that many of these deaths result from unknown exposure (Ciccarone et al., 2017). To mitigate the risks associated with consuming unregulated substances, drug-checking services have been operating for decades, whereby individuals submit drug samples to have the contents identified and analyzed for purity (Barratt, Kowalski, Maier, \& Ritter, 2018; Brunt, 2017; Renfroe, 1986). A 
global review of drug checking services operating in 2017 identified 31 services, three of which were operating in North America: DanceSafe, EcstasyData, and ANKORS (Barratt, Kowalski, et al., 2018). These services reported using reagent tests kits, often in combination with other methodologies, including thin layer chromatography and gas chromatography mass spectrometry (Harper, Powell, \& Pijl, 2017).

In addition to formal drug-checking services, the use of personal reagent test kits appears to be relatively common (i.e., 'informal' drug checking). A recent study estimated that over onefifth $(23 \%)$ of past-year ecstasy consumers in New York City had tested (or had someone test) their ecstasy using a drug testing kit in the past year (Palamar \& Barratt, 2019). Similarly, a study of ecstasy consumers in Australia found that $22 \%$ reported personal use of testing kits (Johnston et al., 2006). However, while numerous studies have explored the prevalence, acceptability, and behavioral outcomes associated with both formal and informal drug checking (e.g., Barratt, Bruno, Ezard, \& Ritter, 2018; Day et al., 2018; Goldman et al., 2019; Measham, 2019), little attention has been devoted to understanding the role and broader experiences of 'drug-checkers' (i.e., people who test their own and/or other people's substances). As such it remains unknown who is testing drugs, the motivations for doing so, and what barriers they may experience. This omission is particularly concerning given that many of these individuals are volunteers, operating in environments which are fraught with political and legal challenges (Barratt, Kowalski, et al., 2018), and may be taking a considerable personal risk in providing such services.

Despite our limited understanding of drug checkers, there is extensive literature regarding volunteerism which may be useful in providing a broader context for this paper. Volunteering is defined by the United Nations (2000) as an act of free will that results in benefits to others. The prevalence of adult volunteering is estimated to be $30 \%$ in the US, $36 \%$ in Australia, and $22 \%$ in Europe (Jenkinson et al., 2013), with Americans volunteering almost 7 billion hours in 2018-an estimated $\$ 167$ billion in economic value (Corporation for National and Community 
Service, 2018). Indeed, volunteerism is a critical resource for many community or non-profit organizations, and considerable attention has been devoted to understanding why people engage in this practice.

However, while motivation to volunteer is a well-researched topic, the models used to explain volunteerism are diverse. This is likely a reflection of the complexity of volunteerism itself-a phenomenon that is not clearly delineated and which spans a wide variety of activities, organizations and sectors (Hustinx, Cnaan, \& Handy, 2010). There are many reasons why individuals volunteer, and these vary considerably across contexts and demographic groups (Stukas, Hoye, Nicholson, Brown, \& Aisbett, 2016), however, most motivations tend to be altruistic in nature-such as to 'give something back' to one's community, or to an organization or charity (Jenkinson et al., 2013). For example, research has identified motivations for volunteer firefighting that are common across sectors (e.g., community safety; community contribution), as well as motivations that are unique to firefighting (e.g., heroism) (Perrott \& Blenkarn, 2015). Furthermore, there are motivational differences across demographic groups, with research showing that female volunteer firefighters are less likely to be motivated by impulsive sensation seeking compared to their male counterparts (Perrott \& Blenkarn, 2015), while younger volunteers were more likely to be motivated by self-oriented perceived benefits compared with older volunteers (McLennan \& Birch, 2008).

Considering this research, we anticipate that although motivations for drug-checking will likely share commonalities with those outlined above, we may also identify motivations unique to drug checkers, and unique to different types of drug checkers. This paper will also make an important contribution in its inclusion of both 'formal' and 'informal' drug-checkers, which we define as being affiliated and not being affiliated with a drug checking organization, respectively. Indeed, one of the limitations of the broader volunteering literature is that it largely focuses on 'formal' volunteering (i.e., volunteering for a formally structured 
organization), with informal volunteering (e.g., helping a neighbor) much less studied. As previously mentioned, formal drug-checking services have been operating for decades; however, the use of personal test kits (i.e., informal drug-checking) is also common, and we argue that both need to be considered. It is thus the aim of this paper to address this research gap by exploring the experiences and motivations of both formal and informal drugcheckers, providing important information regarding the provision of these harm reduction initiatives and identifying potential areas for improvement.

\section{Methods}

A purposive sample of 32 adults was recruited through study flyers on social media and on drug information message board websites frequented by 'psychonauts'. Individuals were also recruited at harm reduction conferences and via referral from other participants. This loose design allowed us to interview a variety of individuals throughout North America with different experiences. To be eligible for this study, individuals must have 1 ) been age $\geq 18,2$ ) speak English, 3) report having tested drugs, and 4) self-identify as being highly experienced or familiar with NPS. A screening was conducted over the phone or in person with the lead investigator to ensure eligibility. Interviews were conducted from July 2015 through July 2018. Those deemed eligible were either interviewed in person or over the phone after providing verbal consent to participate. Interviews were open-ended although some specific topics were discussed with all participants. Interviews were recorded and typically lasted about an hour. Participants were compensated \$50 USD for completing the interview.

Interviews were transcribed by one of the authors and multiple cycles of descriptive coding, recoding, subcoding, and pattern-coding of the transcripts were conducted (Saldaña, 2014) using Atlas.ti version 8 software (Friese, 2018). Coding was conducted in an inductive manner (Miles, Huberman, \& Saldaña, 2014) and code saturation was reached within coding of the first ten interviews, other than some later code definition changes and code-splitting (Hennink, Kaiser, \& Marconi, 2017). A second team member double-coded all transcripts for 
accuracy. Dominant and/or repeated codes were then categorized into themes along with quotations that adequately summarized specific topics and themes in order to form a comprehensive picture. After a consensus was reached regarding classification of codes and themes, quotations in each domain were summarized. The New York University Langone Medical Center institutional review board approved all study methods and a Certificate of Confidentiality was also obtained from the National Institutes of Health to further protect participant confidentiality.

\section{Results}

\subsection{Participant Characteristics}

Sample characteristics are presented in Table 1 . The majority of participants were male $(71.9 \%)$, white $(87.5 \%)$, and resided in the US (90.6\%). The mean age was 27.0 (SD = 5.2; median $=28.0)$ with a range from 18 to 38 . The majority $(56.3 \%)$ of participants reported affiliation with a drug checking organization. Among non-affiliated checkers (43.8\%), 57.1\% $(n=8)$ tested for friends, $21.4 \%(n=3)$ tested only for themselves, and $21.4 \%(n=3)$ identified as people who sell drugs and test for their clients. We did not systematically record the types of drugs tested, but the drugs most commonly mentioned by interviewees were ecstasy and LSD, then ketamine, cocaine, methamphetamine, and 2C-B.

\subsection{Emergent Themes}

Two themes emerged during qualitative analyses: 1) motivations for drug checking, and 2) barriers to drug checking.

\section{Theme 1: Motivations for Drug Checking}

Three overlapping forms of motivation appeared to drive these participants to begin drug testing—self-protection, witnessing of negative drug-related experiences (which led to desire to protect self and others), and protection of others-which we describe as sub-themes. The 
third sub-theme focuses on participants' motivation to protect other people who use drugs (PWUD) from harm in the form of altruism.

\section{Self-Protection}

Many participants interviewed identified as current or former members of dance music scenes, often with a history of drug use experience that influenced them to begin drug checking. Many checkers began testing drugs for themselves after suspecting that their drugs contained adulterants, and this practice often branched into testing for the wider community. For example, this checker used to use ecstasy and deduced that her drugs were impure when the same drug tended to have different effects.

I was questioning, "Why is this different all the time?" I'm getting it from the same person...but it feels different every time... what's going on? So I see there's all these other possibilities of things that could be in here. (Female, Black, age 28)

Even without experiencing adverse effects from drugs being adulterated, feelings of doubt regarding what drug was actually consumed led many participants to start testing drugs for themselves (and others).

A lot of my friends were saying stuff like, "You've just taken MDMA too many times". But I still was just left with this nagging feeling like I don't know. And ultimately I sought out the local chapter and got myself a testing kit. I had already been communicating with them because I wanted to try to see how I could get involved, but then this really sort of pushed me in that direction. And so I met up with one of the chapter heads, he sold me a test kit, I went home, tested it, and it was unmistakable...almost certainly one of those "bath salt" drugs. (Male, White, age 28)

I just saw that a lot of the time, at least from the smaller dealers, that the drugs wouldn't be what you're purchasing. I just thought it was a good idea to always have 
the kits on me. There's a good chance that this won't be what I'm buying, so I should always have the kits and I should test it every time. It's not worth taking a chance. (Male, White, age 21)

Other checkers had negative experiences using drugs (other than merely suspecting adulterants). A couple of checkers, for example, reported unintentional use of NBOMe-a highly potent stimulant with psychedelic properties. These individuals used this compound thinking it was LSD, and the negative experience resulting from unintentional use served as a wakeup call about the likelihood of unknowingly using potentially dangerous adulterants or drug replacements.

Many checkers appear to have begun testing their own drugs (for self-protection), and then later on felt it was important to test others' drugs prior to consumption in order to inform safer use. In fact, the majority (91\%) of participants interviewed in this study reported testing drugs for others-whether or not they were formally affiliated with a drug testing organization.

Although most participants did not describe specific transitions from testing only for themselves to testing for others, this participant explained that she became aware of drug testing, purchased a test kit, but was unable to bring it into a party. As a result, she resorted to purchasing ecstasy from a stranger without having her test kit available. This situation led her to want to volunteer for an organization to ensure that others do not have the same experience.

After that experience with the whole, "Oh, I've been taking this mystery pill, but I couldn't bring the test kit in because they're checking bags," [we] decided that we wanted to volunteer, found a local chapter, and then started volunteering there. (Female, White, age 25)

Witnessing of Adverse Drug Experiences 
Witnessing negative experiences involving drugs also appeared to be a driving factor for many checkers who would later choose to volunteer their services to help others. For example, one checker described having witnessed medical emergencies such as seizures, and people getting rushed to the hospital, from consuming drugs that were believed to be adulterated with more dangerous substances.

Really more than anything it was just seeing other people have bad experiences...It definitely made me look further into it. (Male, White, age 25)

One participant described an event in which a close friend suffered a brain hemorrhage after unintentionally consuming methamphetamine that was sold as MDMA. He explained that this event was the catalyst for him to start drug checking. Another described a negative experience in which his friends purchased pressed pills at a festival that were sold as MDMA, and these pills reportedly led at least one PWUD to experience a seizure. The pills were later tested and the participant reportedly found out that they likely contained a mixture of synthetic cathinones and other stimulants. He further explained that it was this experience that motivated him to test drugs.

These experiences led these participants to test drugs in the future to help themselves and to help others avoid inadvertent consumption of such adulterants.

\section{Protecting Others: Altruism and Public Education}

Regardless of what led these individuals to become drug checkers, most appeared to be motivated by altruism and the desire to increase safety and minimize risks associated with others' drug consumption. We describe their efforts in terms of altruism not only because they act in a selfless manner and donate their time and services, but also because unlike many other volunteers, drug checkers also risk arrest by handling illegal drugs, in order to promote the safety of others. Thus, they essentially place themselves under legal risk to help protect others. 
Many checkers mentioned the desire to educate the public about the importance of drug checking as there is a high prevalence of adulterated drugs in North American drug markets. Desire to educate was often two-fold-to directly educate those about to consume a specific drug (typically through the participants testing drugs in front of the individuals providing them), and to educate PWUD more broadly. Even participants who only tested for themselves often disseminated results, publicly, on social media, in order to inform harm reduction efforts for others. The work of drug checkers is driven primarily by the idea that drug checking allows PWUD to be more aware of the actual contents of the substance they intend to consume, so they believe checking reduces the risk of consuming an unknown substance which could produce untoward or unpleasant side effects.

When we emphasize the importance [of drug checking], people want to do the right thing; they want to do the safer thing because the safer thing is more fun. [We have] all these resources that will keep people from having traumatic or deadly type experiences. (Female, Black, age 28)

Others mentioned that drug checking can also be a useful educational tool even in cases in which a drug has already been ingested. For example, oftentimes, the same batch of a drug will circulate throughout social circles and parties, and misinformation may accompany the drug. Once PWUD learn about the drug's true contents, this information can serve as a preventative measure in case people do not want to consume the drug after they find out its true contents.

Person $\mathrm{X}$ had this. Now we know what this is, we can spread it (information) in that community: "Hey, don't take this." And you will still have time to catch people before they do it. (Male, White, age 30) 
In such situations, information on adulteration may also inform PWUD that they have unknowingly used an adulterant, and such individuals can try to address potential previously unexpected drug reactions.

While most checkers provide direct drug checking services to others, often placing themselves at legal risk for handling potentially illegal drugs, they also typically want to widely disseminate harm reduction messages. They want PWUD to be educated and use in an informed and safe manner, and a major component of their messages tends to be that anyone who uses a synthetic drug should test that drug.

Everyone should get a drug checking kit. I think the more knowledgeable the user, the less likely they are to have these negative experiences. (Female, White, age 35)

\section{Altruism among People Who Sell Drugs}

Another sub-theme was people who sell drugs testing their product to protect their clients. Participants did in fact discuss unscrupulous dealers who knowingly sell adulterated drugs, but we interviewed and learned about others who sell drugs that do look to protect their clientele. In fact, one participant even stated that he started selling to friends in order to make sure people receive quality product.

The whole reason why I got into selling was because I wanted to make sure my friends weren't getting anything that would hurt them, so it started with ecstasy and then with everything found online. I was like the harm reduction drug dealer. I had tested each batch myself and then I would say, "Okay, it's good," and make sure that I could get my seal approved before it would go to anybody else's body because I wanted them to know what to expect-remove that sort of chance of bad things happening. (Male, White, age 26) 
Some other people who sell drugs also promote clients testing their drugs. This person who sells drugs also tests for her friends.

I don't want anyone to have a bad time or possibly get hurt from my product. I want everyone to be happy and be educated and even buy a test kit of their own to make sure. I had a couple of clients who had their own test kit and I encouraged them to test it because it's so important. But also if you have good product it keeps people coming back and you get a good reputation. (Female, White, age 21)

As seen in the quote above, while this seller does display concern about her clients, she does also point out that demonstrating quality product is good for business. Thus, having these multiple reasons for checking leads us to deduce that their motives, unlike the others interviewed, are not purely altruistic. Business motives appear to be common as the participant quoted below also mentioned drug testing as part of his sales approach to selling drugs.

I'll do a Marquis test if I'm just selling a gram of MDMA. I'll test it in front of people the first time, or if there's a new batch, and it will be like "Oh wow"-sort of like a sales thing. But it's important. I want people to know it's legit. (Male, White, age 31)

The person quoted above who sells also reportedly tests drugs on himself when he does not have reagent tests available. For example, he explained that “Even if I don't want to, I'll have a tab of acid just to make sure the kids are okay," to ensure that the LSD he is selling is safe to use.

Others who sell drugs not only test their own supply, but also offer to test products others are selling or using. Below, one participant explains that in one instance he detected synthetic cathinones in ecstasy someone was selling and the other individual continued to sell them anyway. In response, this participant alerted their community. 
So when I sell MDMA, it's more of an altruistic approach in that I don't like people getting bunk shit. I have actually tested something for another dealer. He bought like a good hundred or so pills and asked me to test them for him. I tested them and ended up finding that they were cathinones. I told him he shouldn't sell them....and then later I found out that he was actually still selling them. So I kind of nameshamed him to a pretty large group of people; so of course, that made him quite angry at me, but I mean I guess if you're going to sell bunk shit, assume your reputation is going to be trashed. (Male, White, age 18)

While spreading the word about dealers who knowingly sell adulterated products can also be seen as a form of altruism (especially as this can place the person providing the warning at risk for retaliation), it can also indicate a sense of competition between people who sell. We theorize that such competition between people who sell-regardless of the reason-may lead to decreased adulteration of the products available.

Other participants discussed how some people who sell drugs used testing as marketing tools at festivals and elsewhere.

A lot of dealers actually start to bring their own test kits around with them at the festivals...the last festival I went to, every dealer I asked had a test kit and they tested it for me in front of me. (Male, White, age 21)

However, this participant also warned that testing in front of clients can actually be a form of deceptiveness, and recommends that people who purchase in such situations need to ensure they are purchasing the same batch of the drug being tested.

Say they wanted to sell butylone instead of MDMA. They can have a capsule that they know is MDMA, take a little bit out of that and test it and then give them a fresh one that doesn't have any taken out of it, and that could be a different chemical. So 
typically what l'll do if its capsules or a powder or something, I will purchase the one that was tested. (Male, White, age 21)

\section{Theme 2: Barriers to Drug Checking}

Many checkers discussed barriers to drug checking. Checkers discussed barriers of two types. One barrier was the overarching concern of arrest due to paraphernalia laws, and the other barrier was more direct and at the individual-level, in which venue owners and party promoters denied checkers access to parties due to fear of insurance liability and/or legal repercussions.

\section{Paraphernalia Laws}

A major concern mentioned by a number of checkers_-both affiliated and not affiliated with organizations-was the perception that drug checking kits could be deemed drug paraphernalia. US federal law does not explicitly state that test kits are considered paraphernalia; however, as of 2019 , all but six US state laws specifically mention drug testing equipment as being considered illegal paraphernalia. The five states that do not mention tests being considered illegal paraphernalia are Alaska, Nebraska, New York, South Carolina, and Wyoming. California does consider test kits paraphernalia, but possession is not specifically criminalized. Although laws in each state vary, most specify that testing or analyzing a controlled substance, and/or possessing equipment used or intended to be used to test controlled substances, is considered illegal.

They (test kits) are paraphernalia in most states. They're paraphernalia in the state that I live in, but the whole point of paraphernalia laws is just to have more counts thrown at someone. You have to have something that is actually illicit for it to actually be paraphernalia. (Male, White, age 30) 
Testing kits are usually considered paraphernalia. Unless you're a person that has a professional or academic research reason to possess a testing kit, a testing kit is considered paraphernalia. In lieu of that fact, I do not keep a testing kit on my person. (Male, White, age 22)

Perhaps paradoxically, New York in particular was considered off limits by some checkers due to perceived paraphernalia laws. So for some, fear might not be based on actual specific state-level laws but instead local unwillingness of promoters or venue owners to allow testing.

When I'm with my organization at festivals, people come up to us and ask if we're testing here and ask why we're not testing in New York - the clubs in New York have specific laws where it could be considered drug paraphernalia if someone should die, you get hurt on these drugs, get arrested with them-which is why we can't do it in New York. (Male, White, age 27)

Although a limitation of this study is that we did not specifically ask participants where they reside (with many stating they have tested drugs in multiple states), others mentioned they believed test kits are not illegal to possess. For example, this non-affiliated checker believes that tests are not illegal to possess per se, but they may still in fact be risky to possess, especially as some officers may believe that those possessing test kits may also possess drugs.

They're not illegal to have. I think it's like a gray area. I think it's just frowned upon and if you do have them. I suppose perhaps that's evidence to just search you. Like, "Oh, this person has drug test kits", like he probably has drugs. In the event that I did have drugs on me, if the police or someone is finding the test kits, they're probably finding the drugs, too. That's kind of hand and hand. (Male, White, age 21) 
Others also noted that state laws may vary and/or may be vague, and vague laws may lead some law enforcers to err on the side of enforcement. Thus, as noted above, many checkers expressed concern about the risk of possessing kits because they feared law enforcement officials may link the possession of a testing kit to checkers having direct contact with illegal substances. Therefore, some checkers expressed caution with regard to where they conducted their testing and generally did not carry kits with them. Others also expressed concern about records of purchasing test kits online being used against them as evidence.

I'm overly paranoid my stuff is being watched so if I were to buy something like that (test kits) off eBay or a website, it would be fairly easy for them to keep record of that and it would be tallying up against me...there are a lot of implications with having possession of one of those or a record online to buying one of those. And you can get subpoenaed to have those records released at any moment, like AT\&T or Verizon (US telecommunication providers) can track your location. (Male, White, age 22)

It should be noted that while some checkers expressed concern about test kits potentially being considered paraphernalia, interestingly, checkers did not explicitly note concern about handling drugs to be tested which tend to be illegal. This lack of concern may reflect past or existing drug use status and thus exposure to this risk when handling their own personal drug supply.

The "RAVE Act"

Although many checkers mentioned that they had not heard of anyone being prosecuted for possession of test kits, many were more directly affected by party promoter and venue owner reluctance to allow their services due to fear of fines or even prison sentences. Checkers affiliated with formal drug checking organizations in particular expressed concern over potential legal barriers associated with drug checking at festivals or other events. The 
main concern cited by many formal checkers is the "RAVE Act" (the "Reducing Americans' Vulnerability to Ecstasy Act"). Although this bill in 2002 was never passed, it was reintroduced in US Congress the following year as the Illicit Drug Anti-Proliferation Act which made it illegal to knowingly lease, rent, or use a space for the purpose of distributing or using controlled substances. In response to this Act, party promoters and venue owners fear they would be prosecuted if their venue could be deemed a place where drug consumption was "allowed". According to this Act, property owners or promoters could be fined up to $\$ 250,000$ and face up to 20 years in prison if their parties were deemed places where illegal drug use was taking place.

Checkers commonly cited this law (which they still refer to as the "RAVE Act") as a major barrier to their harm reduction services as such organizations are often not given permission by party promoters or venue owners, because permitting them to operate on-site could be seen as encouraging or allowing drug use. There were also instances mentioned in which drug checking organizations acquire initial approval to set up a booth at a festival, and then the organization is denied access the day of the festival due to fear of legal liability. This checker, for example, cites the "RAVE Act" as the major barrier to drug checking.

Yeah it is only the Rave Act. There's really no other reason. But when it comes to the higher-ups at these events, they have a liability to worry about and their lawyers are intelligently advising them to not allow harm reduction groups into events because quite frankly they could be charged as criminals, their land could be seized and massive fines so I could understand their decision to not allow us. (Male, White, age 29)

According to the checkers, it seemed as though a lot of the fear associated with the "Rave Act" was the uncertainty of whether what they were doing would be deemed as violating this Act. Because it is somewhat unclear in the Act, many venue owners or promoters simply do not want to engage in legal risk. According to one checker, there have not been any 
recorded cases of the Act being used against a venue in recent years. However, the fear appears to remain among promoters and venue owners.

The Rave Act basically gives clearance to producers to be as non-harm reduction focused as possible... and then on the back end claim that they didn't know people were doing drugs at their party, which is how deaths over the last five years have been dealt with, versus the threat that say for example, you perform harm reduction, something happens, and then insurance comes down on you forever. (Male, Other, age 32)

Without having permission to bring test kits into a festival, it was sometimes described as being nearly impossible to sneak test kits in, especially considering the size of tests kits is often larger than small doses or batches of drugs.

Electric Daisy or Ultra-these are kind of unreachable scenes for us. The security is incredibly intense. There are no options for camping where we can kind of sneak around and inform people and of course they won't let us in. l've been to events where the security is just unbelievable-you have three separate check points before you actually get into the music venue. (Male, White, 29)

Checkers therefore appear to be somewhat limited to where they can obtain permission to perform drug checking at public venues. Many expressed that their drug checking was thus largely limited to underground parties or at individual's homes.

We cannot do any drug testing really anywhere ever. The only places we can test are like underground raves that aren't officially put on by promoters. (Male, White, age 23) 
Despite fear of law enforcement repercussions among checkers, promoters, and venue owners, drug checking does still occur at many festivals, or for festival attendees, but often through loopholes or through checking in an informal manner. One formal checker mentioned that people can request an appointment on their website and she will then go test a person's drugs at his or her place before a party. Sometimes a drug checking organization might be permitted to oversee a harm reduction booth at a party, but drug checking is often not approved. In such circumstances, checkers may have to oversee implementation of testing more secretly in other areas. Or, when a drug checking organization is not given permission to set up a booth at a party, checkers may meet PWUD inside or outside of the party (or send peers to do so) to help them test their drugs. Thus, checking can still be conducted when official permission or access is denied, but in a less visible and less formal manner.

Usually what we do is we tell people that we have a friend that can go and test with them. That kind of thing very much varies on location. (Female, White, age 19)

It should be noted that even some participants who are not formally affiliated with a drug checking organization also are interested in testing drugs for festival attendees and find ways to test drugs inside festivals.

There are friends of mine who do promoting that have been able to get me in (to test drugs), just more under the hush-hush. (Male, White, age 25)

One checker also pointed out that PWUD can simply order the test kits online themselves and receive them within four days. In such cases, PWUD can test their drugs on their own time-given they know how. Thus, promoting self-testing or teaching people to self-test prior to the party can help ensure that testing is less needed at the party location.

Even when there aren't formal drug checking booths set up at parties, checkers expressed that many attendees do still manage to bring in testing kits, so the barrier of lack of 
permission for formal testing does not necessarily prevent all patrons from testing drugs themselves at parties. Sometimes test kits are even distributed among patrons at parties. However, when drug checking must remain hidden, it can occur in a somewhat dangerous manner as caution needs to be taken when handling such chemicals.

Who knows under what conditions people may be testing-you know it could be a bathroom stall in the club or this dark corner. Some people take [test kits] to festivals and they're testing it in weird conditions; they're not at home with your gloves, your goggles...whatever. (Female, Black, age 28)

Some checkers mentioned that it is easier to bring test kits into festivals that have campsites, even though vehicles are usually searched on their way into the sites.

These are large events with mild fencing. It's so easy for someone to just walk 100 yards away from security and throw something over the fence. We sneak 2000+ baseball size substance test kits into every event that we attend and we do it with ease. It really is not difficult so if we are able to do that with 8 or 10 duffle bags (Male, White, age 29)

Despite the "RAVE Act" being a common barrier to drug testing, some checkers did note that law enforcement officials or security at some festivals appear to have more permissive attitudes towards checking, and in some cases security officials are said to have actually helped distribute test kits.

There are a lot of local law enforcement [officials] who probably know that this is going on. I think that a lot of more progressive law enforcement [officials] do understand that this is potentially reducing the amount of hospitalizations and deaths that happen at these events and are kind of knowingly looking the other way. I bet a lot of them don't explicitly know that we're doing this or just don't want to know. (Male, White, age 28) 
We see a lot of people a little bit lower on the totem pole of gate security agents, the people that wander through the campsites, allowing the test kits to happen, allowing our people to distribute them. We even run into groups of security guards that have gone so far as to buy their own test kits and to go out there and allow people to use them behind closed doors or behind closed tent flaps (Male, White, age 29)

\subsection{Differences Between Affiliated and Non-Affiliated Checkers}

We observed many commonalities between checkers who reported being affiliated with drug checking organizations and those who are not affiliated. The themes discussed above apply to both affiliated and non-affiliated checkers, although unaffiliated participants were less likely to discuss the "RAVE Act" as being an obstacle for testing.

We deduce that both affiliated and unaffiliated checkers began drug checking for similar reasons-as discussed above-for self-protection and for altruistic reasons (to protect others). Checkers tended to begin testing for themselves and/or for friends. A portion of this group goes on to join or even start their own drug checking organization (or a chapter within an organization). It appears that most affiliated checkers still test drugs for friends, but these individuals tend to discuss their testing in terms of checking at parties or festivals, where they often conduct a high volume of tests within a day or weekend. Affiliated individuals also commonly discussed their testing experiences in terms of "we", which suggests they feel like they collectively belong to a group or organization and are contributing to a cause.

Most affiliated checkers discussed their experiences in terms of checking at booths or tents at parties. Unaffiliated checkers, however, tended to discuss their informal experience more in depth. The testing situations discussed by unaffiliated checkers at times was much different from checkers who conduct most of their testing, formally, at booths at festivals, and have strangers who use drugs approach them to test. Many of these informal checkers discussed their willingness to go to people who need testing. 
I usually test for my friends. Like if I know my friends are buying anything, I will test it for them or I will ask them to let me do it. Even if it's not for me, l'd rather keep my friends alive. (Male, White, age 22)

I have a testing kit, you know. l'll test whatever for anybody. If you want to give me a little bit, l'll test it, or I will tell you to buy your own test kit and give you the website. (Female, White, age 38)

If you ever need anything tested, all they need to do is they need to come to me, and I can go ahead and I can get the kit and I can come to them. We can meet up at a location of their choice, for their own safety and peace of mind, or I can direct them towards an individual and I can facilitate a meeting between them and the individual will have the testing kit. (Male, White, age 22)

Of note, many of these unaffiliated checkers test drugs at or near college campuses, which is a much different "scene" than raves and dance festivals.

I have said you know if anybody needs drugs checked just put them in contact with me. And then it spread like wildfire from there because it's a college town. Everybody wants to get high. (Male, White, age 25)

It should be noted that these independent drug checkers also self-fund their own drug tests, so they not only spend time testing for others, but they use their own money to purchase the tests. Although it should be noted that sometimes these independent checkers do in fact accept small amounts of money or drug product for testing for others.

But that was up until I was able to save up the money to be able to afford my own drug testing kit, which have been doing well for a long time now. (Male, White, age 25) 
Some members of drug checking organizations in particular tended to also be active regarding wider dissemination of drug test findings. For example, some more formal checkers post alerts on social media warning that either certain drugs (e.g., pressed pills) or drugs in certain areas have been found to be adulterated or replaced with drugs such as "bath salts". Some individuals at these organizations also disseminate drug test findings from websites such as EcstasyData.org (based on information provided by a laboratory and not their own tests) to further alert communities. Unaffiliated participants typically did not report such dissemination of results. Although it should be noted that one checker who also described himself as a psychonaut mentioned that he posts his test results on public message boards.

Every substance that I do, including the new lysergamides, I post up all the reagent tests, I post up my personal reviews of it, so that's just sort of my involvement in the community of these new novel substances. (Male, White, age 25)

Finally, one more difference between affiliated and unaffiliated individuals was direct challenging of drug dealers who sell adulterated products. Some affiliated checkers mentioned that when they detect adulterants in a sample at a festival, the PWUD may approach the seller and ask for a refund (which checkers often recommend against as they fear the PWUD can then place themselves in a different type of danger). However, only nonaffiliated checkers described directly challenging people who sell adulterated drugs.

But that's also why when I do encounter drug dealers, I'm like, "Bring a kit with you". If you're gonna be purchasing a substance, why don't you just bring your kit with you? And if they won't let you test your substance then maybe that's not who you should be buying your substances from. (Female, White, age 24)

So last year there was somebody selling what he said was $2 \mathrm{C}-\mathrm{B}$, but it was on blotter paper and 2C-B isn't active enough in that small of a dose. So, I asked him if we 
could test it and immediately came up as NBOMe so we told him that what he was selling was really dangerous and if somebody is injured or dies because of what they take and it's traced back to him, he could be in a lot of trouble. As far as I know, he stopped selling that stuff. (Male, White, age 22)

In sum, we deduced some differences in practices between those affiliated and those not affiliated with drug testing organizations; however, it appears that these differences in practices were still driven by similar motivations.

\section{Discussion}

Drug checking services have been operating for decades (Barratt, Kowalski, et al., 2018; Brunt, 2017; Renfroe, 1986), and personal testing kits are now readily accessible and commonly utilized (Johnston et al., 2006; Palamar \& Barratt, 2019); however, there remains a dearth of information regarding the experiences of people who test their own and/or other people's drugs. This study appears to be the first to explore the experiences of people who test drugs. The themes identified in this analysis (motivations and barriers) provide important information regarding the provision and improvement of these harm reduction initiatives.

Given that one of the key objectives of drug checking is to inform consumers about the content and purity of substances so that they can make a more informed decision about their drug use (Brunt, 2017), it was not surprising that 'self-protection' was one of the main motivations endorsed by participants. For some participants the desire to engage in drug checking emerged from an increasing realization that they were consuming adulterated substances, while for others it was an adverse event (either their own, or someone else's) which motivated them to initiate drug checking. These motivations are consistent with other research, which demonstrates that exposure to negative experiences can motivate engagement in harm reduction initiatives. For example, a study of family members of people 
who use opioids found that witnessing an overdose was a significant motivation for engaging in naloxone training and overdose education programs (Bagley et al., 2015).

With regard to testing other people's drugs, participants appeared to be motivated by altruism and the desire to increase safety and minimize risks associated with others' drug consumption. While there is no comparable work among other samples of drug checkers, studies in other fields support similar findings. For example, a study of the social supply of ecstasy through friendship networks found that one of the motivations for social dealing (i.e., supplying to friends for no cash profit) was to help friends minimize risk (Bright \& Sutherland, 2017). That is, ecstasy consumers were willing to risk purchasing and supplying illegal substances in order to provide a potential health benefit to their peers. Similarly, our participants were willing to provide illegal or legally ambiguous services in order to provide harm reduction services for their peers. Challenging the conceptualization of PWUD as 'irresponsible' and 'untrustworthy' (Lancaster, Seear, \& Treloar, 2015), these findings show that many PWUD are committed to improving the safety of themselves and their peers, particularly in the absence of adequate government policies.

Furthermore, we identified a small group of 'altruistic people who sell drugs' who tested their products prior to sale, partly to protect their clients and partly as a 'sales pitch' to generate repeat business. Although these motivations were not always purely altruistic, distributing unadulterated drugs still helps reduce potential harm to people who use, and may be a modern method of acquiring 'trust' from clients - similar to dealers who give free samples or allow potential clients to try test samples of drugs (Coomber, 2006). Indeed, trust is an important, and mutually beneficial, element of illicit drug transactions. For the customer, trust helps increase the odds that the product received is of good quality, with recent research indicating that: i) many consumers have a high level of trust in their dealers; and ii) using a trusted and reliable drug dealer is often viewed as a harm reduction strategy by consumers (Bardwell, Boyd, Arredondo, McNeil, \& Kerr, 2019; Carroll, Marshall, Rich, \& Green, 2017; 
McKnight \& Des Jarlais, 2018; Soukup-Baljak, Greer, Amlani, Sampson, \& Buxton, 2015; Taylor \& Potter, 2013).

With this is mind, it has been suggested that rather than perpetuating the narrative of 'evil' and 'amoral drug dealers' (Coomber, 2006), we could engage these individuals and encourage them to test their supplies and develop communication strategies to more accurately inform PWUD of drug contents and avert risks associated with using them (Bardwell et al., 2019). Our findings suggest that drug-checking has been adopted by some people who sell drugs; however, it remains unclear how the results are being interpreted and communicated to consumers. Some of the participants spoke as if testing made drug use completely safe (e.g., "remove that chance of bad things happening"). Overly simplistic messages, such as "okay, it's good", raise multiple concerns. Firstly, given that all or most of our participants reported use of reagent test kits, test results may be inaccurate or incomplete. Reagent test kits can only identify the presence (or absence) of a single substance (multiple reagent test kits are needed to identify the presence of more than one substance), they cannot test for all substances (Cuypers, Bonneure, \& Tytgat, 2016), nor can they quantify the presence of substances. To date, there do not appear to be any studies which have validated the interpretation and communication of personal test kit results, and this is an area that would benefit from future research. Secondly, even if the most accurate technology is used to test drugs, only part of the risk is removed by drug checking, given that consuming drugs with known contents and strength can still result in harms. These nuances may be lost in exchanges where checkers are overly confidence in their practices.

While our findings support the broader literature regarding motivation to volunteer, which has consistently identified altruism as a key motivation, we acknowledge that altruism remains a contested concept. At its core, altruism "captures a concern for others that is not linked to a concern for oneself" (Rutherford, 2010, pg. 1033); however, there are many different types 
of, and theoretical viewpoints on, altruism (Feigin, Owens, \& Goodyear-Smith, 2014). One of the most prominent divisions relates to the motivation underpinning altruism. For example, altruistic acts without a conscious expectation of reward are considered 'true' (i.e., selfless) altruism, while acts with the conscious or unconscious expectation of reward are considered pseudo-altruistic (i.e., selfishly-motivated). Recently, it has been argued that theories of altruism should allow for the possibility of co-existing motivational drives (egoistic and altruistic) (Feigin et al., 2014), and we would argue that this holds true for our 'altruistic people who sell drugs'. That is, while people who sell drugs were testing their products to protect their clients-with one individual entering into drug dealing with the explicit aim to make sure their peers received quality product that "wouldn't hurt them"-they also engaged in drug testing to ensure their clients 'keep coming back' and to acquire a good reputation.

The second theme to emerge from this study focuses on the barriers associated with drug checking. The provision of drug-checking services remains fraught with political and legal problems (Barratt, Kowalski, et al., 2018), and hence it was not surprising that the main barriers identified by our study revolved around legality. Specifically, participants were concerned about arrest due to paraphernalia laws, and were commonly denied permission to set up drug checking services at events due to concerns about the 'RAVE Act'. Although to our knowledge no major music festival organizer appears to have been prosecuted under this Act (Mohr, 2018), our findings indicate that festival organizers and event promoters continue to fear that the presence of drug checking services will imply prior knowledge of drug use occurring at their events, thus making them potentially liable and subject to fines and/or criminal sanctions. Subsequently, participants most commonly provided these services at underground parties or at private homes, thus limiting the scope of these important harm reduction initiatives. Combined, these findings suggest that it could be beneficial to public health if the 'RAVE Act' and/or drug paraphernalia legislation was amended to exempt drug-checking services and personal testing kits, respectively (Mohr, 2018). Indeed, the health of PWUD at dance parties may be better protected if festival 
organizers, event promoters and drug-checkers did not have to fear fines and/or criminal prosecution for providing potentially life-saving services.

\subsection{Limitations}

This study was exploratory, with a relatively small sample size, and hence our findings cannot be considered representative of all drug checkers. Furthermore, all our participants were recruited from North America, and complex cultural and regulatory differences mean that motivations and barriers experienced by drug-checkers could vary considerably across countries and states. We also did not specifically ask participants about their area (e.g., state) of residence so we were unable to determine how local policies affect drug checking or perception of legality of drug checking where they live. Although it should be noted that many participants also tested drugs at festivals throughout various states. Our sample was also primarily drawn from dance music and clubbing scenes where ecstasy/MDMA was the main drug type that checkers were testing. Most interviews were conducted before the availability of fentanyl test kits which is likely why discussion of fentanyl adulteration was lacking. However, although most checking among participants in this sample focused only on party drugs, we believe these findings also have implications for fentanyl testing. Extrapolation of our findings to different settings, for example, where heroin is being checked for the presence of fentanyl and related analogs (Krieger et al., 2018), may be limited. Finally, qualitative research involves interpretation by the research team. Themes were coded and processed by two independent individuals; however, it is possible that different themes or sub-themes, could emerge from different investigators. As such, our findings cannot be considered definitive, but rather offer a foundation from which future research can be built.

\section{Conclusion}


Drug checkers in North America seek to educate themselves and other PWUD about the risk of exposure to unexpected substance types. They do so by volunteering a service for their peers where samples of one's drug supplies can be tested and psychoactive substances identified. Information from this activity is then often widely distributed among networks of PWUD. Some people who sell drugs also utilize drug testing kits as part of their service, which is framed in terms of safety as well as a marketing tool. While illicit drugs have no formal quality control because they are illegal, perhaps convincing people who sell drugs to test their products and only sell unadulterated products can help "clean up" the drug market. Public health agencies, drug checkers and even consumers can inform people who sell drugs about the potential benefits of drug checking and convince them to test their own drugs. Likewise, people who use drugs can test drugs themselves or only purchase from people who test their drugs (and sell unadulterated product). Further education about the limits of reagent testing may help avoid the circulation of overconfident safety messaging and help promote a dual message that while avoiding adulterated drugs is desirable, consumption of tested drugs is not without risk: it can and does also result in harm.

Barriers to drug checking included concern of arrest due to drug testing kits being considered drug paraphernalia and, in relation to formal drug checking organizations, lack of access to venues due to fear of insurance liability and/or legal repercussions. Repeal of legislation that deems drug testing kits to be drug paraphernalia would help to relieve some of these barriers, as would the reform or repeal of the Illicit Drug Anti-Proliferation Act (Mohr, 2018). These policy reforms would not only help formal drug checking organizations operate in the US more openly, but they would also allow drug checking organizations to upgrade their technology to enable more accurate and comprehensive identification and quantification of substances, in a similar fashion to European drug checking services (Barratt, Kowalski, et at., 2018). That is, while checking is conducted in a legal gray zone, checkers are unable to apply for funding to purchase more expensive equipment and nor can they insure (or even transport) such equipment if operating clandestinely. Legislative 
changes could help ensure that these potentially life-saving services can be provided without fear of fines and/or criminal prosecution. 


\section{Acknowledgements}

Research reported in this publication was supported by the National Institute on Drug Abuse of the National Institutes of Health under Award Numbers K01DA038800 (PI: Palamar), R01DA044207 (PI: Palamar), and P30DA011041 (PI: Hagan). The National Drug and Alcohol Research Centre is supported by funding from the Australian Government under the Drug and Alcohol Program. The content is solely the responsibility of the authors and does not necessarily represent the official views of the funders. 


\section{References}

Australian Criminal Intelligence Commission. (2019). Illicit Drug Data Report 2017-18. Canberra: Australian Criminal Intelligence Commission

Bagley, S. M., Peterson, J., Cheng, D. M., Jose, C., Quinn, E., O'Connor, P. G., \& Walley, A. Y. (2015). Overdose Education and Naloxone Rescue Kits for Family Members of Individuals Who Use Opioids: Characteristics, Motivations, and Naloxone Use. Substance Abuse, 36(2), 149-154. doi:10.1080/08897077.2014.989352

Bardwell, G., Boyd, J., Arredondo, J., McNeil, R., \& Kerr, T. (2019). Trusting the source: The potential role of drug dealers in reducing drug-related harms via drug checking. Drug and Alcohol Dependence, 1-6. doi:https://doi.org/10.1016/j.drugalcdep.2019.01.035

Barratt, M. J., Bruno, R., Ezard, N., \& Ritter, A. (2018). Pill testing or drug checking in Australia: Acceptability of service design features. Drug Alcohol Rev, 37(2), 226-236. doi:10.1111/dar.12576

Barratt, M. J., Kowalski, M., Maier, L. J., \& Ritter, A. (2018). Global review of drug checking services operating in 2017. Retrieved from Sydney: https://ndarc.med.unsw.edu.au/sites/default/files/ndarc/resources/Global\%20review\% 20of\%20drug\%20checking\%20services\%20operating\%20in\%202017.pdf

Bright, D. A., \& Sutherland, R. (2017). "Just Doing a Favor for a Friend": The Social Supply of Ecstasy Through Friendship Networks. 47(3), 492-504. doi:10.1177/0022042617704004

Brunt, T. M. (2017). Drug checking as a harm reduction tool for recreational drug users: opportunities and challenges Retrieved from Luxembourg: http://www.emcdda.europa.eu/system/files/attachments/6339/EuropeanResponsesG uide2017 BackgroundPaper-Drug-checking-harm-reduction 0.pdf

Brunt, T. M., Nagy, C., Bücheli, A., Martins, D., Ugarte, M., Beduwe, C., \& Ventura Vilamala, M. (2017). Drug testing in Europe: monitoring results of the Trans European Drug Information (TEDI) project. Drug Test Anal, 9(2), 188-198. doi:10.1002/dta.1954

Carroll, J. J., Marshall, B. D. L., Rich, J. D., \& Green, T. C. (2017). Exposure to fentanylcontaminated heroin and overdose risk among illicit opioid users in Rhode Island: A mixed methods study. International Journal of Drug Policy, 46, 136-145. doi:https://doi.org/10.1016/j.drugpo.2017.05.023

Ciccarone D, Ondocsin J, \& Mars SG. (2017). Heroin uncertainties: Exploring users' perceptions of fentanyl-adulterated and -substituted 'heroin'. International Journal of Drug Policy, 46:146-155. doi: 10.1016/j.drugpo.2017.06.004.

Cole, C., Jones, L., McVeigh, J., Kicman, A., Syed, Q., \& Bellis, M. (2011). Adulterants in illicit drugs: a review of empirical evidence. 3(2), 89-96. doi:10.1002/dta.220

Coomber, R. (2006). Pusher myths: re-situating the drug dealer. Free Association Books, London, UK.

Corporation for National and Community Service. (2018). Volunteering in America. Retrieved from https://www.nationalservice.gov/serve/via

Cuypers, E., Bonneure, A.-J., \& Tytgat, J. (2016). The use of presumptive color tests for new psychoactive substances. 8(1), 136-140. doi:10.1002/dta.1847

Day, N., Criss, J., Griffiths, B., Gujral, S. K., John-Leader, F., Johnston, J., \& Pit, S. (2018). Music festival attendees' illicit drug use, knowledge and practices regarding drug content and purity: a cross-sectional survey. Harm reduction journal, 15(1), 1. doi:10.1186/s12954-017-0205-7

European Monitoring Centre for Drugs and Drug Addiction. (2019). European Drug Report 2019: Trends and Developments. Luxembourg: European Monitoring Centre for Drugs and Drug Addiction

Feigin, S., Owens, G., \& Goodyear-Smith, F. (2014). Theories of human altruism: a systematic review. Journal of Psychiatry and Brain Functions, 1(5). 
Friese, S. (2018). ATLAS.ti 8 Windows - User Manual. Retrieved from http://downloads.atlasti.com/docs/manual/atlasti_v8_manual_en.pdf

Goldman, J. E., Waye, K. M., Periera, K. A., Krieger, M. S., Yedinak, J. L., \& Marshall, B. D. L. (2019). Perspectives on rapid fentanyl test strips as a harm reduction practice among young adults who use drugs: a qualitative study. Harm reduction journal, 16(1), 3-3. doi:10.1186/s12954-018-0276-0

Harper, L., Powell, J., \& Pijl, E. M. J. H. R. J. (2017). An overview of forensic drug testing methods and their suitability for harm reduction point-of-care services. 14(1), 52. doi:10.1186/s12954-017-0179-5

Hayner, G. N. (2002). MDMA Misrepresentation: An Unresolved Problem for Ecstasy Users. J Psychoactive Drugs, 34(2), 195-198. doi:10.1080/02791072.2002.10399953

Hennink, M. M., Kaiser, B. N., \& Marconi, V. C. (2017). Code Saturation Versus Meaning Saturation: How Many Interviews Are Enough? Qual Health Res, 27(4), 591-608. doi:10.1177/1049732316665344

Hustinx, L., Cnaan, R. A., \& Handy, F. (2010). Navigating Theories of Volunteering: A Hybrid Map for a Complex Phenomenon. 40(4), 410-434. doi:10.1111/j.14685914.2010.00439.x

Jenkinson, C. E., Dickens, A. P., Jones, K., Thompson-Coon, J., Taylor, R. S., Rogers, M., . . . Richards, S. H. (2013). Is volunteering a public health intervention? A systematic review and meta-analysis of the health and survival of volunteers. BMC Public Health, 13(1), 773. doi:10.1186/1471-2458-13-773

Johnston, J., Barratt, M. J., Fry, C. L., Kinner, S., Stoové, M., Degenhardt, L., . . Bruno, R. (2006). A survey of regular ecstasy users' knowledge and practices around determining pill content and purity: Implications for policy and practice. International Journal of Drug Policy, $17(6), \quad$ 464-472. doi:https://doi.org/10.1016/j.drugpo.2006.03.008

Krieger, M.S., Goedel, W.C., Buxton, J.A., Lysyshyn, M., Bernstein, E., Sherman, S.G., Rich, J.D., Hadland, S.E., Green, T.C., \& Marshall, B.D.L. (2018). Use of rapid fentanyl test strips among young adults who use drugs. International Journal on Drug Policy. 61:52-58. doi: 10.1016/j.drugpo.2018.09.009.

Lancaster, K., Seear, K., \& Treloar, C. (2015). Laws prohibiting peer distribution of injecting equipment in Australia: A critical analysis of their effects. International Journal of Drug Policy, 26(12), 1198-1206. doi:https://doi.org/10.1016/j.drugpo.2015.05.014

McKnight, C., \& Des Jarlais, D. C. (2018). Being "hooked up" during a sharp increase in the availability of illicitly manufactured fentanyl: Adaptations of drug using practices among people who use drugs (PWUD) in New York City. International Journal of Drug Policy, 60, 82-88. doi:https://doi.org/10.1016/j.drugpo.2018.08.004

McLennan, J., \& Birch, A. (2008). Why Would You Do It? Age and Motivation to Become a Fire Service Volunteer. The Australian and New Zealand Journal of Organisational Psychology, 1, 7-11. doi:10.1375/ajop.1.1.7

Measham, F. C. (2019). Drug safety testing, disposals and dealing in an English field: Exploring the operational and behavioural outcomes of the UK's first onsite 'drug checking' service. Int J Drug Policy, 67, 102-107. doi:10.1016/j.drugpo.2018.11.001

Miles, M. B., Huberman, A. M., \& Saldaña, J. (2014). Qualitative Data Analysis: A Methods Sourcebook, Third Edition. Retrieved from Thousand Oaks, CA:

Mohr, R. (2018). Preventing Drug-Related Deaths at Music Festivals: Why the "Rave" Act Should be Amended to Provide an Exception for Harm Reduction Service. ChicagoKent Law Review, 93(3), 943-969.

Mohr, A. L. A., Friscia, M., Yeakel, J. K., \& Logan, B. K. (2018). Use of synthetic stimulants and hallucinogens in a cohort of electronic dance music festival attendees. Forensic Science International, 282, 168-178. doi:10.1016/j.forsciint.2017.11.017

Morelato, M., Beavis, A., Tahtouh, M., Ribaux, O., Kirkbride, P., \& Roux, C. (2014). The use of organic and inorganic impurities found in MDMA police seizures in a drug intelligence perspective. Sci Justice, 54(1), 32-41. doi:10.1016/j.scijus.2013.08.006 
Morgan, J. P. (1982). The Jamaica Ginger Paralysis. JAMA, 248(15), 1864-1867. doi:10.1001/jama.1982.03330150050022 \%J JAMA

Palamar, J. J., \& Barratt, M. J. (2019). Prevalence of reagent test-kit use and perceptions of purity among ecstasy users in an electronic dance music scene in New York City. 38(1), 42-49. doi:10.1111/dar.12882

Palamar, J. J., Salomone, A., Gerace, E., Di Corcia, D., Vincenti, M., \& Cleland, C. M. (2017). Hair testing to assess both known and unknown use of drugs amongst ecstasy users in the electronic dance music scene. International Journal of Drug Policy, 48, 91-98. doi: 10.1016/j.drugpo.2017.07.010

Perrott, S., \& Blenkarn, B. (2015). Motivation, sensation seeking, and the recruitment of volunteer firefighters. International Journal of Emergency Services, 4(2), 242-257.

Rutherford, A. (2010). Get by with a little help from my friends: A recent history of charitable organisations in economic theory. The European Journal of the History of Economic Thought, 17(4), 1031-1046. doi:10.1080/09672560903434489

Renfroe, C. L. (1986). MDMA on the street: Analysis Anonymous. J Psychoactive Drugs, 18(4), 363-369. doi:10.1080/02791072.1986.10472371

Saldaña, J. (2014). The Coding Manual for Qualitative Researchers, 2nd edition. Los Angeles, CA: SAGE

Scholl, L., Seth, P., Kariisa, M., Wilson, N., \& Baldwin, G. (2018). Drug and opioid-involved overdose deaths - United States, 2013-2017. Morbidity and Mortality Weekly Report, 67(5152):1419-1427. doi: 10.15585/mmwr.mm675152e1.

Soukup-Baljak, Y., Greer, A. M., Amlani, A., Sampson, O., \& Buxton, J. A. (2015). Drug quality assessment practices and communication of drug alerts among people who use drugs. International Journal of Drug Policy, 26(12), 1251-1257. doi:https://doi.org/10.1016/j.drugpo.2015.06.006

Stukas, A. A., Hoye, R., Nicholson, M., Brown, K. M., \& Aisbett, L. (2016). Motivations to Volunteer and Their Associations With Volunteers' Well-Being. 45(1), 112-132. doi:10.1177/0899764014561122

Taylor, M., \& Potter, G. R. (2013). From "Social Supply" to "Real Dealing":Drift, Friendship, and Trust in Drug-Dealing Careers. 43(4), 392-406. doi:10.1177/0022042612474974

United Nations. (2000). United Nations Volunteers Programme: Preparatory Committee for the Special Session of the General Assembly on the implementation of the outcome of the world summit for social development and further initiatives. Volunteering and social development. Vienna: United Nations

United Nations Office on Drugs and Crime. (2019). Word Drug Report 2019. Vienna: United Nations Office on Drugs and Crime

Verweij, A. (1992). Impurities in Illicit Drug Preparation: 3, 4-(Methylenedioxy) amphetamine and 3, 4-(Methylenedioxy) methamphetamine. Forensic Science Review, 4, 137-137. 